\title{
Nutrition education strategy with father involvement to improve infant feeding practices in Kisumu, Kenya.
}

\author{
Lynette $\mathrm{AD}^{\mathbf{1}^{*}}$, Beatrice $\mathrm{NK}^{\mathbf{1}}$, Florence $\mathrm{K}^{\mathbf{1}}$ \\ Department of Human Nutrition Sciences, Jomo Kenyatta University of Agriculture and Technology, Kenya
}

\begin{abstract}
Fathers' support is an essential component in ensuring success in improving the infant feeding practices. A nutrition education intervention strategy was employed targeting the fathers in the intervention group with information on breastfeeding and complementary feeding over the one year research period. A total of 290 father-and-mothers to be paired were recruited. Study participants were randomized into either intervention group ( $n=145$ pairs) or control group ( $n=145$ pairs). The fathers completed a pre-tested structured questionnaire that explored their knowledge of breastfeeding pre and post-intervention. Kisumu County was selected because of the high infant mortality rate (105 deaths per 1,000 live births) in the region. Our results did not show any statistically significant differences in the knowledge level on breastfeeding of fathers in the intervention and control group pre-intervention based on odds ratio test of the following indicators: initiation of breastfeeding within an hour of birth $(p=0.44)$, exclusive breastfeeding for 6 months $(p=0.79)$ and breastfeeding for 2 years and beyond $(p=0.90)$. However, statistically, significant differences were observed post-intervention as follows: initiation of breastfeeding within an hour of birth $(p=0.02)$, exclusive breastfeeding for 6 months $(p=<0.01)$ and breastfeeding for 2 years and beyond $(p=0.01)$. A Wilcoxon signed-rank test showed a statistically significant change in knowledge of the fathers in the intervention group after receiving the nutrition education sessions $(Z=-10.181, p=0.000)$. Therefore, we conclude from our study that nutrition education targeting the fathers increases their knowledge on breastfeeding and this presents a good opportunity to enhance their support towards the mother, ultimately contributing to improved infants feeding practices.
\end{abstract}

Keywords: Father involvement, Nutrition education, Kisumu, Kenya, Infant feeding.

\section{Introduction}

Evidence of effective ways to assist fathers in their supportive role with breastfeeding is scarce with limited published examples within the Kenyan context [1]. The exclusive breastfeeding rate in Kenya is at $61 \%$, and this is below the WHO recommendations of $90 \%$ [2]. Effective strategies to support exclusive breastfeeding are required. Behavior changes strategies for the adoption of recommended breastfeeding practices focus on improving the knowledge gaps of mothers with little attention to the influence of fathers in the improved infant feeding practices [3]. A mother's decision to initiate and continue breastfeeding has been strongly linked to the father's support and influence [4]. Fathers can make a difference in the rate of the infant feeding practices, but they need information in order to make a difference [5,6]. Engagement of fathers by educating them on breastfeeding and men's group activities can greatly improve infant feeding and health behaviors [7].

A randomized controlled trial was done in Italy in which fathers went through a two-hour pre-natal education session on infant care as well as breastfeeding assistance to their partner. The result was a $33 \%$ difference in breastfeeding initiation among women whose partners had attended the class, in comparison with the controls [8]. Another study in Italy, also found that teaching fathers how to prevent and manage the most common lactation difficulties had a marked, positive impact on breastfeeding continuation. Few (15\%) mothers whose partners had been simply told about the benefits of breastfeeding were still breastfeeding at age six months; but when the men were individually given information for just 40 minutes on management of common problems (for example pain and discomfort, fear that baby isn't 'getting enough' and breastfeeding-issues when mum returns to work) the percentage of mothers still breastfeeding at age six months was $25 \%$. The impact was particularly strong among women who had reported difficulties with lactation (4.5\% vs. $24 \%)$ [9].

In Kenya, most community programs seeking to improve the well-being of women and children target young mothers and their children [1]. Few initiatives in the country have engaged men in efforts to improve the infant feeding [1]. A pilot project done in Western Kenya, engaged men through nutrition education on the infant and young child feeding practices through group training of men facilitators using a training manual targeted to men [10].

Based on the outcomes in the studies in Italy, we hypothesize that the study in the Kenyan context would result in positive outcomes on the infant feeding when fathers are reached with information on breastfeeding and complementary feeding. The objective of our study was to assess the impact of using nutrition 
education to mothers and fathers as a strategy to improve the infant feeding. This is because studies have shown that there has been less attention on male involvement in the infant feeding thus the timeliness of our study.

\section{Methodology}

\section{Development of nutrition education intervention material}

Nutrition education materials on recommended breastfeeding and complementary feeding practices with specific information tailored for fathers emphasizing their unique and vital role in the success of breastfeeding and complementary feeding were developed. The education material was based on the UNICEF integrated infant and young child feeding counseling, a training course released in 2012 [11] and Infant and Young Child Feeding and Gender manual prepared by the Infant \& Young Child Nutrition (IYCN) Project for the men's groups activities [10].

\section{Study setting and study design}

This study was conducted in Kisumu East Sub County, Kisumu County, Kenya. The county has 6 sub-counties namely: Kisumu East, Kisumu West, Kisumu North, Nyando, Muhoroni, and Nyakach. Kisumu East Sub County has a total area of 135.9 $\mathrm{Km}^{2}$ and a population of 150,124 [2]. The study participants were recruited from the Kisumu County hospital in Kisumu East Sub County. It is a Level 5 Hospital providing services such as antenatal, basic emergency, obstetric care, curative, out-patient and in-patient services, family planning and growth monitoring among others.

The study was a randomized controlled trial (RCT) where participants were randomized into either an intervention (receiving education on infant feeding) or a control group (not receiving education on infants feeding). The target population was fathers (husbands of women who are recruited at 6 months pregnant at the time of recruitment into the study) and motherinfant pair.

\section{Sampling}

Calculation of the sample size was done using the formula by Johnson et al. [12]. The study expected an effect size of $20 \%$ based on the intervention of nutrition education targeting both father and mother. This was because the outcome was expected to be better than that of a previous study done in Italy which conducted the nutrition education sessions post-natal thus, not much time for father-mother pairs to make an early informed decision. Our study conducted the nutrition education sessions pre-natal with the expectation that father-mother pairs will be in a position to make an early informed decision which could influence right from early initiation of breastfeeding. The study in Italy had considered a difference in the effect of the intervention of $15 \%$ and used a standard deviation of 0.58 [9].

$n=\frac{2\left(Z_{\alpha}+Z_{1-\beta}\right)^{2} \sigma^{2}}{\Delta^{2}}$

Where:

n- Estimated sample size
$\mathbf{Z}_{\alpha}$ level of significance (set at $0.95 \alpha=0.05=1.96$ ); the $\mathbf{Z}_{1-\beta}$ power of the study $(80 \%)$

$\boldsymbol{\Sigma}$ standard deviation (estimated at 0.58 )

$\Delta$ difference in the effect of interventions which is required (estimated at $20 \%$ based on previous studies) [9]

Hence

$n=\frac{2(1.96+0.8416)^{2}(0.58)^{2}}{(0.20)^{2}}$

$\mathrm{n}=132$.

Considering a $10 \%$ attrition rate, the total sample size was 145 which was the number considered for the intervention group which would receive the nutrition education intervention.

Overall, 290 father-mother pairs were enrolled in the study. Ten participants were recruited per day from those who agreed to take part in the study from a daily attendance to the antenatal clinic of 75 pregnant women with an estimate of a third being 6 months pregnant according to the hospital profile. Contacts of the recruited study participants were taken and used to follow up on the fathers for their consent to participate in the study.

All the women who were 6 months pregnant between January and April 2016 and attending the antenatal clinic at Kisumu County Hospital were eligible for inclusion in the study. Once recruited, each woman provided the telephone contact of the father-to-be. The latter were then invited to participate in the study. A meeting between the father-mother pairs and the study team took place within 2 weeks of the first contact. The pair were asked to pick one among pre-numbered papers which randomly put them in either the intervention or control group.

\section{Delivery of the nutrition education intervention}

Nutrition education intervention was given to the intervention group. The mothers to be who had been recruited during the antenatal clinic visit gave their phone contacts and follow up phone calls were made to the fathers to be. Consent was sought from the fathers and mothers to participate in the study. Those who consented to participate in the study together with their partners were invited to Kisumu County Hospital for nutrition education session prenatally on breastfeeding and complementary feeding which lasted 4 hours. The education sessions were delivered once to the intervention group. The language of communication during the intervention delivery was Dholuo, a local language that could be easily understood by the study participants. Nutrition education was provided in the form of group facilitated sessions to the intervention group. The study participants started with a pre-test of knowledge on infants feeding to assess their knowledge levels on the same. Questions on breastfeeding on demand, exclusive breastfeeding, good positioning and attachment to the breast, benefits of breastfeeding, food consistency and frequency during complementary feeding were read to the study participants and responses recorded on questionnaires. A post-test was also conducted with similar questions in the pre-test after the nutrition education sessions [11]. At the end of the nutrition education sessions, a leaflet with the main points of the session was provided to the participants.

Direct (teacher-directed), interactive (discussion, sharing) and 
experiential (learner-centered, activity-oriented) instructional strategies were used in the education sessions. Demonstrations on positioning and attachment of the baby to the breast, expressing of breast milk and food demonstrations were done for maternal nutrition and complementary feeding to show how to ensure consistency, frequency, and variety per different age groups. Role plays were done to demonstrate how the father can rub the back of the mother to stimulate milk production, how a father can encourage the mother to build her confidence in breastfeeding, cup feeding for the expressed breast milk and playing with the baby to stimulate growth and development. Case studies were done on how to manage common breast conditions such as engorgement, inverted nipples, and flat nipples. Procedures for preparing nutritious meals based on promoted recipes were described during the sessions. The recipes were based on the seven food groups recommended for children [6]. Fathers were also enlightened on the supportive role they can play such as ensuring there is adequate nutritious food for the mother during pregnancy and lactation, supporting the mother during delivery by encouraging her to put the baby to the breast within an hour of birth, rubbing the mothers back to stimulate milk production, holding the baby for the mother to get rest, cup feeding the baby on expressed breast milk, providing adequate nutritious food for the baby during complementary feeding, helping in feeding the baby, helping in taking care of older children and doing household chores.

The study considered the social cognitive theory for behavior change. Father involvement through nutrition education was expected to result in adequate knowledge on infants feeding by the father and fathers' know-how of the supportive role they can play in infant feeding. This would result in positive environmental influence to the mother and improved adherence to recommended infant feeding practices at the household level. Hence leading to an improved nutritional status of the infant expected to contribute ultimately to the reduction in malnutrition rates and morbidity patterns.

\section{Data management and analysis}

Data was field edited, coded and entered into computer software. SPSS version 21 was used to analyze the quantitative data for both descriptive and inferential statistics. Frequencies and associations were done on knowledge on breastfeeding including breast milk as first feed, initiation of breastfeeding, exclusive breastfeeding and continued breastfeeding in the intervention and control group pre-intervention and postintervention. Data from the intervention and control group was analyzed based on the pre-test and post-test questionnaires with the median of the pre-test and post-test scores being recorded and measures of significance between the two scores recorded.

\section{Results}

A total of 290 father-mother pairs were analyzed at baseline with 145 pairs in the intervention group and control group respectively. At 3 months post-intervention; in the intervention group, 8 father-mother pairs were lost to follow up due to relocation ( 5 pairs), the death of the child (1 pair) and separation of the couple ( 2 pairs). In the control group, 3 father-mother pairs were lost to follow up due to relocation ( 2 pairs) and death of the child (1 pair). The dropout rate does not influence the current results as the sampling used considered an attrition rate of $10 \%$.

\section{Fathers' knowledge on infant feeding}

At baseline, the odds of fathers in the intervention group being more knowledgeable about breastfeeding than fathers in the control group were measured. The result was not statistically significant (Table 1).

Post-intervention when comparing fathers in the intervention group compared with the control group, fathers in the intervention group were 1.6 times more likely to be knowledgeable that breastfeeding should be initiated within an hour of birth $(\mathrm{OR}=1.61 ; \% \mathrm{CI}: 1.07-2.42 ; p=0.02), 1.7$ times more likely to be knowledgeable that breastfeeding should continue until 2 years and beyond $(\mathrm{OR}=1.72 ; \% \mathrm{CI}: 1.14-2.59 ; p=0.01)$ and were twice likely to be more knowledgeable on exclusive breastfeeding (OR=2.01; \% CI: $1.27-3.19 ; p<0.01)$ (Table 2).

\section{Pre-test and post-test results for the intervention group}

Based on the box plot in Figure 1, the center for post-test scores is much higher than the center for pre-test scores with more spread being observed in the pre-test scores than the post-test scores. The results of the pre-test scores appear not to be symmetrically distributed while post-test scores are symmetrically distributed with outliers. A Wilcoxon signed-rank test showed that nutrition education involving the fathers elicited a statistically significant change in knowledge of the fathers on infant feeding $(Z=$ $10.181, \mathrm{p}=0.000$ ). Indeed, median pre-test score was $50 \%$ while the post-test score was $83.3 \%$.

\section{Assessment and rating of the nutrition education sessions}

From Table 2, it was clear that the major $(66.9 \%, n=97)$ source of information on infant feeding for mothers in the intervention group prior to the study, was the health facility during antenatal clinic visits. The majority $(76.6 \%, n=111)$ of the fathers reported that they did not have any source of information on infant feeding. A high $(92.1 \%, \mathrm{n}=134)$ proportion of fathers from the intervention group felt that they had received new information from the nutrition education session offered by the research team. Less than half $(40 \%, n=58)$ of the mothers

Table 1. The odds ratio for father's knowledge about breastfeeding pre-intervention.

\begin{tabular}{|c|c|c|c|c|}
\hline Characteristics & Control ( $\mathrm{N}=145)$ & Intervention ( $\mathrm{N}=145)$ & Odds ratio $(95 \% \mathrm{Cl})$ & p-value \\
\hline \multicolumn{5}{|c|}{ Knowledge about breast-feeding } \\
\hline Breast milk first feed & $95.2(138)$ & $94.5(137)$ & $1.01(0.73-1.42)$ & 0.93 \\
\hline Breastfeed within 1 hour & $52.3(75)$ & $56.5(82)$ & $0.84(0.54-1.31)$ & 0.44 \\
\hline Breastfeed for 2 years & $55.2(80)$ & $55.5(79)$ & $1.03(0.67-1.59)$ & 0.90 \\
\hline EBF & $40.1(58)$ & $38.6(56)$ & $1.07(0.64-1.81)$ & 0.79 \\
\hline
\end{tabular}

Note: EBF: Exclusive Breastfeeding; The level of significance based on odds ratio is $p$-value $<0.05$. The figures in the bracket are ' $n$ ' with $\mathrm{N}=145$ in the control group and $\mathrm{N}=145$ in the intervention group. The figures outside bracket represent the $\%$ within the group. 
felt that they had received new information from the nutrition education session. New knowledge received as reported by the intervention group was in the areas of paternal support that can be provided during infant feeding, how breastfeeding works, expressing of breast milk, and preparation of complementary foods (ensuring frequency, amount, thickness, variety, and hygiene).

In terms of time allotment, the majority of the fathers $(73.4 \%$, $\mathrm{n}=106)$ felt that the 4 hours allocated was about right while $58.5 \%(\mathrm{n}=85)$ of the mothers shared the same view. Over half $(55.3 \%, n=80)$ of the fathers were of the opinion that the content taught was extremely relevant in assisting participants in practicing recommended infant feeding practices. A third $(54.5 \%, \mathrm{n}=79)$ of the mothers agreed to the opinion of the content being extremely relevant in assisting in the practice of recommended infant feeding practice. In terms of teaching quality, over three-quarters $(75 \%, n=109)$ of the fathers felt that it was very high with a similarly high percentage of the mothers holding the same opinion $(77 \%, n=112)$. The teaching method which encompassed a lot of discussions, demonstrations and role plays was termed by a majority of the fathers $67 \%(n=97)$ and mothers $74.8 \%(n=108)$ as appropriate. On the other hand, the interest level of the participants in the contents taught was rated as very high by both the father and mother at $79.3 \%$ $(n=115)$ and $81 \%(n=117)$, respectively.

However, a chi-square test was performed and no relationship was found between gender and rating on time allocation, $\mathrm{X}^{2}$ $(2, \mathrm{~N}=290)=5.10, \mathrm{p}=.77$; gender and rating on relevance of the content, $\mathrm{X}^{2}(2, \mathrm{~N}=290)=4.39, \mathrm{p}=.68$; gender and quality of teaching, $\mathrm{X}^{2}(2, \mathrm{~N}=290)=2.13, \mathrm{p}=0.89$ and gender and interest level in the session, $X^{2}(2, N=290)=1.01, p=0.61$ (Table 3).

\section{Discussion}

Studies have shown that a lack of knowledge can influence the success and duration of breastfeeding [13-15]. The fathers' knowledge level about breastfeeding was considered key for supporting the mothers' decisions and actions [8]. Similar to our study at baseline, a cross-sectional study was done in Brazil that assessed paternal breastfeeding knowledge during pregnancy. The findings showed a lack of breastfeeding knowledge among the fathers with a specific knowledge deficit on how to manage breastfeeding complications, promote lactation and assure a proper latch [16]. Our findings indicated an increase in knowledge on infant feeding by fathers when fathers were exposed to nutrition education. This, therefore, is a pointer that nutrition education strategies that involve fathers could positively impact on their knowledge levels.

In our study, the nutrition education sessions took place prenatal within a hospital setting to fathers and mother in the intervention group. The exclusive breastfeeding rates were higher in the group in which both the mothers and the fathers were educated about exclusive breastfeeding than in the groups in which there was no education and in which only the mothers were educated[14]. Recommendations from another study are that nutrition education to the father should take place during the antenatal period and the baby's first quarter [17]. A trial offering fathers a 2-hour breastfeeding antenatal class and postnatal support found that breastfeeding levels were increased

Table 2. Fathers' knowledge about breastfeeding practices post-intervention (3 months).

\begin{tabular}{|c|c|c|c|c|}
\hline Characteristic & Control $(\mathrm{N}=142)$ & Intervention $(\mathrm{N}=137)$ & Odd ratio $(95 \% \mathrm{Cl})$ & $\mathrm{p}$-value \\
\hline \multicolumn{5}{|c|}{ Knowledge about breastfeeding } \\
\hline Breast milk first feed & $97.8(139)$ & $94.5(137)$ & $1.11(0.79-1.57)$ & 0.54 \\
\hline Breastfeed within 1 hour & $57.7(82)$ & $75.4(104)$ & $1.61(1.07-2.42)$ & $0.02^{*}$ \\
\hline Breastfeed for 2 years & $56.3(80)$ & $71.7(99)$ & $1.72(1.14-2.59)$ & $0.01^{*}$ \\
\hline EBF & $43.9(62)$ & $63.7(88)$ & $2.01(1.27-3.19)$ & $0.00^{* *}$ \\
\hline
\end{tabular}

Note: EBF: Exclusive Breastfeeding; The level of significance based on odds ratio is $\mathrm{p}$-value $<0.05$. ${ }^{*}$ used for $\mathrm{P}<0.05$ and ${ }^{* *}$ used for $\mathrm{P}<0.01$. The figures in the bracket are ' $n$ ' with $\mathrm{N}=142$ in the control group \& $\mathrm{N}=137$ in the intervention group. The figures outside bracket represent the $\%$ within the group.

Table 3. Rating for the nutrition education sessions by the intervention group.

\begin{tabular}{|c|c|c|c|}
\hline Ratings for nutrition education sessions & Father \% (n) & Mother \% (n) & p-value \\
\hline \multicolumn{4}{|c|}{ Time allocation } \\
\hline Too short & $20.7(30)$ & $27.6(40)$ & \multirow{3}{*}{0.77} \\
\hline About right & $73.4(106)$ & $58.5(85)$ & \\
\hline Too long & $5.9(9)$ & $13.9(20)$ & \\
\hline \multicolumn{4}{|c|}{ The relevance of the content } \\
\hline Extremely relevant & $55.3(80)$ & $54.5(79)$ & \multirow{3}{*}{0.68} \\
\hline Somewhat relevant & $37.9(55)$ & $40.7(59)$ & \\
\hline Not very relevant & $6.8(10)$ & $4.8(7)$ & \\
\hline \multicolumn{4}{|c|}{ Quality of the teaching } \\
\hline Very high & $75.0(109)$ & $77.1(112)$ & \multirow{3}{*}{0.89} \\
\hline Somewhat high & $21.4(31)$ & $18.6(27)$ & \\
\hline Somewhat low & $3.6(5)$ & $4.3(6)$ & \\
\hline \multicolumn{4}{|c|}{ Teaching methods used } \\
\hline Appropriate & $67.0(97)$ & $74.8(108)$ & \multirow[b]{2}{*}{0.89} \\
\hline Need adjustment & $33.0(48)$ & $25.2(37)$ & \\
\hline \multicolumn{4}{|c|}{ The interest level of the participants in the session } \\
\hline Very high & $79.3(115)$ & $81.0(117)$ & \multirow{3}{*}{061} \\
\hline Somewhat high & $13.8(20)$ & $15.2(22)$ & \\
\hline Somewhat low & $6.9(10)$ & $3.8(6)$ & \\
\hline \multicolumn{4}{|c|}{ Chi-square results where $p>0.05$ indicates not statistically significant, $N=290, n=145$ for both the control and the intervention groups. } \\
\hline
\end{tabular}




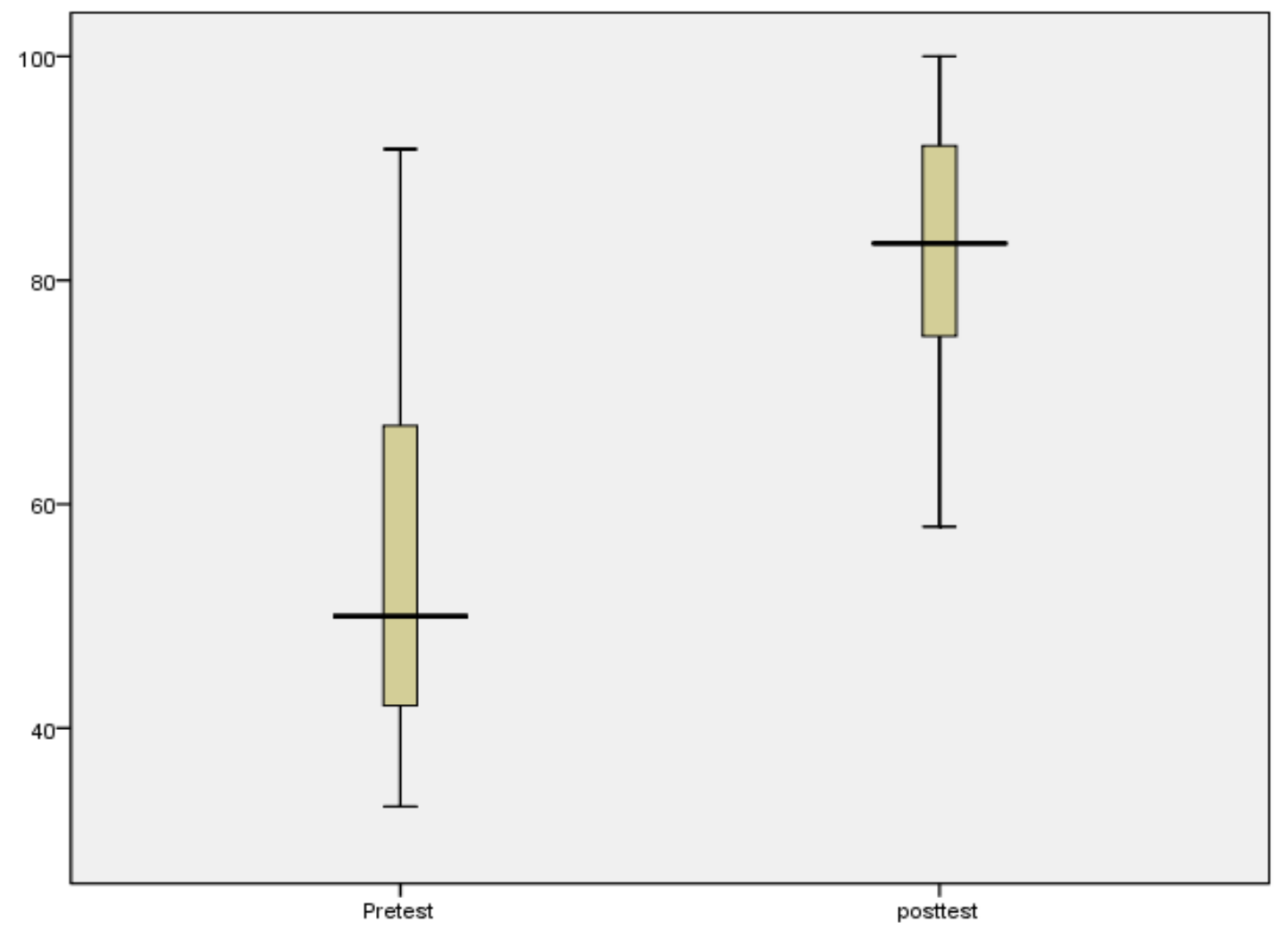

Figure 1. Box plot on the pre-test and post-test results for fathers in the intervention group.

at 6 weeks post-partum $[18,19]$. Another study that invited fathers to attend breastfeeding antenatal classes alongside their partner increased the duration of exclusive, but not overall, breastfeeding [14].

Health professionals can utilize opportunities such as antenatal visits to educate the fathers on infant feeding which can be a cost-effective channel of reaching the fathers. Majority of the fathers reported receiving new information and seeing relevance on what was taught. This can demystify the notion that childcare is only a woman's concern and look for avenues to reach out to the fathers as well.

The main limitation of our study is that though it draws upon literature from other countries, the analysis does not take into account possible cultural similarities and diversities with regards to what constitutes a father's support. Hence further research that includes testing an international model of the fathers' support relating to infants feeding which could build on and expand the findings reported here, paying particular attention to the cost-benefit analysis to investigate the cost-effectiveness of the classroom-based nutrition education strategy and to ascertain the feasibility of implementing it in various settings. In addition, the study sample was selected from a hospital setting which has possible differences with the community settings as antenatal clinic visits are not optimal in Kenya which could have affected the representation of the population sampled.

\section{Conclusion}

Nutrition education to the fathers can improve their knowledge on infant feeding and equip them with skills on providing a supportive role. The fathers revealed a lack of knowledge on infants feeding prior to the intervention which points to the importance of healthcare professionals to consider avenues of reaching out to the fathers in prenatal infant feeding promotion interventions. The time allotted for the nutrition education session can be split to cover the 4-hour session during different contact points during the antenatal period.

\section{References}

1. Thuita FM. Infant and young child feeding practices in Kenya: A review of the literature. Nairobi, Kenya.: PATH 2008.

2. Kenya National Bureau of Statistics (KNBS) and ICF Macro. 2014 Kenya Demographic and Health Survey 2010-2014. Kenya: Calverton, Maryland: KNBS and ICF Macro.

3. Mclnnes RJ, HoddinotP, Britten J, et al. Significant others, situations and infant feeding behavior change processes: A serial qualitative interview study. BioMedical Central. 2013;13(1)- 114.

4. Britton CM. Support for breastfeeding mothers. Cochrane Database of Systematic Reviews. 2007;1-64.

5. Kenosi M, Hawkes, CP, Dempsey EM, et al. Are fathers underused advocates for breastfeeding? Irish Medical Journal. 2011; 104: 313-315.

6. Tohotoa J, Maycock B, Hauck YL, et al. Dads make a difference: An exploratory study of parental support for breastfeeding in Perth, Western Australia. International Breastfeeding Journal. 2009; 4:15.

7. Sloand E, Astone NM, Gebrian B. The impact of fathers' clubs on child health in rural Haiti. American Journal of Public Health. 2009;100(2): 201-204.

8. Wolfberg AJ, Michels KB, Shields W, et al. Dads as breastfeeding advocates: results from a randomized controlled trial of an educational intervention. American Journal of Obstetrics and Gynecology. 2004; 19(3):708-712. 
9. Pisacane A, Continisio GI, Aldinucci M, et al. A controlled trial of the father's role in breastfeeding promotion. Pediatrics. 2005; 116:e494-e498.

10. Martin S, Mukuria A, Maero P. Engaging men to increase support for optimal infant feeding in Western Kenya. Kenya: USAID 2009.

11. UNICEF. The Community Infant and Young Child Feeding Counselling Package. New York, USA: UNICEF 2012.

12. Johnson RA, Dean W, Wichern SS. Practical biostatistical methods. Belmont: Duxbury Press, 1995.

13. Hauck YL, Hall WA, Jones C. Prevalence, selfefficacy and perceptions of conflicting advice and selfmanagement: effects of a breastfeeding journal. J Adv Nurs. 2007;57(3):306-317.

14. Susin LR, Giugliani ER. The inclusion of fathers in an intervention to promote breastfeeding: impact on breastfeeding rates. Journal of Human Lactation. 2008; 24(4): 386-392.
15. Fairbank L, O’Meara S, Renfrew M, et al. A systematic review to evaluate the effectiveness of interventions to promote the initiation of breastfeeding. Health Technol Assess. 2000; 4(25):1-69.

16. Cardoso A, Silva AP, Marin H. Fathers' To Be' Knowledge about breastfeeding. International Journal of Pregnancy and Child birth. 2017; 2(3):00023.

17. Arora S, McJunkin C, Wehrer J, et al. Major factors influencing breastfeeding rates: mother's perception of father's attitude and milk supply. Pediatrics. 2000; 106(5): e67.

18. Maycock B, Binns CW, Dhaliwal S, et al. Education and support for fathers improve breastfeeding rates: a randomized controlled trial. Journal of Human Lactation. 2013; 29:484-490.

19. Kenya National Bureau of Statistics (NNBS) and ICF Macro. A 2010 Kenya Demographic and Health Survey from 2008-2009. Kenya: Calverton, Maryland: KNBS and ICF Macro 2010.

\section{*Correspondence to:}

Lynette AD

Department of Human Nutrition Sciences

Jomo Kenyatta University of Agriculture and Technology

Kenya

Tel: +254725681922

Fax : 254-067-52164/52030

E-mail: linetaoko@yahoo.com 